\title{
Infraestructuras de confianza y sostenibilidad. Una mirada a las relaciones entre sostenibilidad y estandarización en la política internacional de producción cafetera
}

\author{
Infrastructures of trust and sustainability. \\ An exploration to the relationship between sustainability and \\ standardization in the International Coffee production policy
}

Derly Yohanna Sanchez-Vargas ${ }^{1}$

\begin{abstract}
Resumen
Este artículo presenta el aporte de los estudios sociales de la ciencia y la tecnología a la comprensión de las configuraciones sociales y técnicas que introducen los estándares de sostenibilidad en la producción cafetera. Se hace énfasis en la importancia de entender las infraestructuras materiales y sociales que sostienen, a menudo de forma invisible, prácticas y órdenes institucionales. En el caso de la producción agrícola, una agenda política global que promueve la sostenibilidad ha atraído un conjunto de actores que prometen producción más sostenible a través de su estandarización. Este artículo explora el potencial analítico y empírico del estudio de los procesos de estandarización de café, especialmente en el caso de los sellos de sostenibilidad.
\end{abstract}

Palabras clave: estandarización; café; sostenibilidad; infraestructuras

Tipología: Artículo de investigación

Recibido: $11 / 03 / 2019$

Evaluado: $15 / 05 / 2019$

Aceptado: 06/06/2019

Disponible en línea: 09/06/2019

Como citar este artículo: Sanchez-Vargas, Derly Y. Infraestructuras de confianza y sostenibilidad. Una mirada a las relaciones entre sostenibilidad y estandarización en la política internacional de producción cafetera. Jangwa Pana, 18 (2), 214-231. Doi: DOI: http://dx.doi.org/10.21676/16574923.3007

\footnotetext{
${ }^{1}$ Candidata a doctorado en Work, Technology and Organization, Universidad de Lancaster, Reino Unido. Investigadora grupo de Estudios Sociales de la Ciencia y la Tecnología, Universidad Nacional de Colombia. Docente del programa de sociología de la Universidad del Rosario, Bogotá, Colombia. ORCID ID: 0000-0003-0126-4879 Correo electrónico: dysanchezv@gmail.com
} 


\begin{abstract}
This paper discusses some contributions from Social Studies of Science and Technology (STS) in understanding the socio-technical configurations that sustainability standards bring on coffee production. STS addresses the importance of invisible social and material infrastructures institutional and practical orders. This also explores the analytical and empirical landscape of sustainability standards on coffee production.
\end{abstract}

Keywords: standardization; coffee; sustainability; infrastructures

\section{Introducción}

$\mathrm{E}_{\mathrm{d} e}^{\mathrm{n}}$ febrero de 2019, la Federación Nacional de Cafeteros de Colombia anunció la posibilidad de retirar del mercado el café tipo exportación. Los precios bajos, la volatilidad del mercado, el aumento de los costos de producción y el cambio climático son algunos de los factores que han afectado el futuro de la producción de café para inversionistas, gobiernos y productores en el sur global. Sin embargo, este problema no es nuevo. Desde finales de la década de 1990, el sector cafetero a nivel mundial ha experimentado una situación de inestabilidad y precariedad. Ante tal escenario, los estándares de sostenibilidad se han venido presentando durante los últimos 15 años como una solución "efectiva" $y$ "confiable" para múltiples agentes, prometiendo acceso a mercados globales a aquellos productores que demuestren su cumplimiento. Este artículo explora las infraestructuras que producen dichos estándares y la tensión que generan con la producción "tradicional" de café.

Los estándares de sostenibilidad del café, tales como Rainforest Alliance, Fairtrade, Organic Certificated, Birdfriendly y UTZ, son normas internacionales creadas e impulsadas por Organizaciones No Gubernamentales (ONG) u organizaciones de consumidores o empresas privadas que, luego de auditoría y certificación, prometen acceso a cadenas de valor agregado (es decir, que se pague un precio extra al acordado nacional e internacionalmente), a formas de protección de la naturaleza y a prácticas de trabajo "justo" y formalizado en la fase de producción agrícola de café. Los estándares (tanto las normas como las prácticas de auditoría y certificación) conforman infraestructuras de estandarización fuertemente mediadas por conocimientos administrativos, ambientales y sociales. Estos estándares se manifiestan materialmente en actas de visita, listas de chequeo, formatos de visita y etiquetas de trazabilidad.

Según la Federación Nacional de Cafeteros (en adelante FNC), para el año 2017 se registraron 236 mil fincas que producen bajo estándares de sostenibilidad en Colombia. Esto, en términos de la FNC, quiere decir que pertenecen a "algún programa de cafés sostenibles" y equivalen a un $39 \%$ de las hectáreas cultivadas en café del país. Pertenecer a algún programa de sostenibilidad significa, en este contexto, que los productores cafeteros cumplen requerimientos específicos, y de forma voluntaria están relacionados con formas de producción más amigable con el medio ambiente (mantenimiento de corredores biológicos, no uso de pesticidas prohibidos, protección de fuentes hídricas) y mejores prácticas laborales, los cuales son auditados y certificados a través de evaluaciones rigurosas por organizaciones certificadoras.

Este artículo hace parte de un programa de investigación más amplio que explora cómo los estándares de sostenibilidad producen 
infraestructuras materiales y regulatorias que posibilitan la materialización de mercados de café sostenibles en Colombia. La pregunta clave que orienta este programa es ¿cómo los "aspectos sociales, ambientales y económicos" de dichos estándares se configuran narrativa y materialmente en algunas localidades específicas relevantes para su producción/aplicación? Por localidades de producción/aplicación se entienden aquellas organizaciones globales y nacionales que promulgan los estándares, las organizaciones que los producen, las agencias de auditoría y certificación, y las fincas cafeteras que acogen los sistemas de certificación. Este es un esfuerzo por seguir y articular la multiplicidad de los estándares de sostenibilidad.

Estos últimos se configuran mediante conocimientos de expertos de las ciencias sociales, como el derecho internacional, los estudios sobre desarrollo, la administración y las ciencias socioambientales, ensamblando formas particulares de entender qué es lo sostenible en las localidades de las fincas cafeteras. Como todo proceso de estandarización, los sellos dejan por fuera otra serie de relaciones ecológicas y sociales dentro de la producción de café. Por eso, esta investigación recurre a las sensibilidades ofrecidas por los estudios sociales de la ciencia y la tecnología (STS) sobre la infraestructura y los estándares (Leigh Star y Lampland, 2009; Bowker and Leigh Star, 2009), la antropología de las conexiones globales (Tsing, 2005) y los estudios sobre la performatividad de los conocimientos tecnocientíficos y sus métodos de producción y desarrollo (Woolgar et. al, 2009). Este conjunto heterogéneo de herramientas conceptuales me permite explorar cómo los estándares de sostenibilidad producen natur-culturas específicas (Haraway, 2003), que algunas veces se adaptan o entran en contradicción y tensión con prácticas y ontologías locales.

Este artículo, sin embargo, tiene también un fin más modesto: reseñar los aportes clave de la literatura y esbozar un conjunto de problemas importantes que emergen en el estudio de los procesos de estandarización en la producción de café, a partir de una revisión de la política y "la regulación" internacional. Para tal fin, reconstruye aspectos clave del posicionamiento discursivo de los estándares de sostenibilidad en la política ambiental y cafetera internacional, mirando tanto las conferencias de sostenibilidad como los documentos técnicos producidos por la Organización Internacional del Café (de aquí en adelante ICO en inglés u OIC en español) y la Federación Nacional de Cafeteros de Colombia (FNC).

A continuación, se discute el proceso mediante el cual los estándares de sostenibilidad se posicionan en el discurso de la política social y económica de los organismos internacionales encargados de la protección del ambiente y en los que estos estándares representan la herramienta adecuada para la transformación de los mercados de café. A partir de un análisis de las políticas y los marcos regulatorios que dan forma a los estándares de sostenibilidad FairTrade y Rainforest Alliance, se muestran diferentes aspectos de las infraestructuras normativas mediante las cuales pretenden gobernar lo social y lo ambiental en la producción cafetera global y local. Finalmente, cierro con una discusión sobre las formas como los estándares de sostenibilidad responden a una concepción de desarrollo sostenible que promete una articulación armónica entre la protección de la naturaleza y el desarrollo del capitalismo global en términos de comercio internacional. Dicha armonización entre economía y sostenibilidad configura un discurso holístico por parte de las certificadoras internacionales, donde sostenibilidad ambiental y económica son un solo proceso.

\section{La estandarización como formas sociotécnicas de organización}

Las normas y los procesos de estandarización se han convertido en temas de investigación para las ciencias sociales en los últimos años 
(Bain and Selfa, 2017; Bain 2014; Bain y Hatanaka, 2010, Bush, 2011; Lampland y Leigh Star, 2009; Renard, 2010; Loconto, 2014, Van der Kamp, 2012a). Disciplinas como la sociología, los estudios de organización, las ciencias de la información y los estudios de ciencia y tecnología se han acercado al conjunto de documentos, criterios y reglas de clasificación, a menudo invisibles, que posibilitan el curso "normal" de organizaciones, mercados $\mathrm{y}$, en general, de la vida social y económica.

Los STS han entendido los estándares y las prácticas de estandarización como elementos clave en la producción de conocimiento tecnocientífico (Leigh Star y Bowker, 1999), es decir, como dispositivos constitutivos en la distribución y la coordinación, vía sociotécnica, de formas específicas de órdenes sociales, que incorporan políticas de conocimiento y organización. En ese sentido, se muestra, por ejemplo, cómo gran parte del trabajo tecnocientífico consiste en la generación de procedimientos estandarizados que terminan en la multiplicación de las interdependencias, entre el conocimiento producido y las prácticas de clasificación y organización de dicho conocimiento (Mackenzie et al, 2013, Thevenot, 2009).

Así mismo, el estudio de estándares en los estudios sociales de la ciencia se ha desarrollado en relación con la problematización de prácticas de clasificación y cuantificación, mirando las infraestructuras materiales-discursivas que las sostienen $\mathrm{y}$, sobre todo, mostrando las consecuencias sociales de su producción y uso. Estos trabajos han abordado los estándares como dispositivos, clave en la producción de orden y coordinación de prácticas distribuidas entre diferentes mundos sociales o comunidades de práctica, temporalidades y localidades (Bowker y Star, 1999). Los estándares materializan cuerpos de conocimiento disciplinar como el medio ambiental, la salud, la agroalimentación y la administración de procedimientos, normas y formas de organización y desempeño. Estos estudios sobre todo se han enfocado en el establecimiento de regímenes biomédicos (Timmermans y Berg, 2010; Timmermans y Epstein, 2010); más ampliamente, regímenes de mercado y organización (Brunsson, et al., 2000), y políticas agroalimentarias globales (Busch, 2010).

En el caso particular de los estándares de sostenibilidad, Van der Kamp (2012a) argumenta que los estándares son dispositivos que configuran el mercado de lo "sostenible", particularmente el de lo "orgánico", en formas materiales y cotidianas particulares. Los estándares, dice Van der Kamp (2012a), se entienden como dispositivos de mercado que son constantemente reescritos en las prácticas de producción agrícola en las fincas; de certificación y auditoría en las agencias certificadoras; y de mercadeo. Contrario a lo que se piensa, el estándar no es estático, sino que cambia a medida que se mueve.

Otros trabajos han explorado las relaciones entre los estándares de sostenibilidad y los mercados desde la perspectiva de los consumidores, describiendo las situaciones en las que perciben el valor diferencial de la certificación y las economías morales en las que están integrados (Maertens, 2016; Pinto et al., 2014; Barham y Weber, 2012).

Otra mirada sobre los estándares de sostenibilidad en la producción agrícola se interesa en su economía política. Estos estándares se generan y se articulan a formas neoliberales de gobernanza contemporánea, particularmente como formas tripartitas de gobierno de la producción de comida: consumidores, certificadores y agentes privados (grandes superficies, cadenas de comida entre otros) (Bain, et al. 2010, Hakanata, 2010). En esa misma línea, se ha explorado críticamente la relación entre gobernanza tripartita y conocimiento tecnocientífico (Bush y Loconto, 2010), y la constitución del campo organizativo, y saber experto, de la 
sostenibilidad. En particular, Loconto y Fouilleux (2014) analizan el caso de Iseal Alliance, una organización global que reúne vía membresía a varias organizaciones estandarizadoras de sostenibilidad, mostrando cómo su rol es el de legitimar actores privados o no gubernamentales en la regulación de la sostenibilidad global. Finalmente, estos trabajos reflejan un interés en cómo los aspectos sociales de la sostenibilidad son representados y escritos en los estándares de sostenibilidad. Bain (2010, 2014), por ejemplo, explora cómo los estándares de GlobalGAP estructuran un mercado laboral flexible y feminizado en zonas rurales en Chile.

Por su lado, la literatura colombiana sobre estándares de producción cafetera en Colombia se ha enfocado en estudiar cómo impacta su aplicación a los procesos y dinámicas sociales y productivas de las fincas cafeteras. Trabajos como el de Rueda y Lamibini (2012) estudian el uso de Rainforest Alliance como forma de certificación en fincas en Santander. Autores como Rueda y Lambini (2012) y Gulh (2009) reconocen que las fincas cafeteras manifiestan mejoras relacionadas con la presencia e interés por la biodiversidad en los predios, el uso controlado de agroquímicos y una percepción positiva de los cafeteros en cuanto al precio de su café, como resultado de su decisión de ingresar a estos programas.

A continuación, se presenta una mirada histórica al surgimiento de estos estándares en los discursos de política ambiental y cafetera internacional.

\section{Sostenibilidad, café y el camino de la estandarización}

Los orígenes del concepto de sostenibilidad, como asunto político, podrían remontarse a la década de 1970. En Estocolmo (1972) se organizaron las primeras reuniones para discutir la idea de poner límite al crecimiento económico desbordado, que luego de la segunda guerra mundial había tomado lugar en los países industrializados. Sin embargo, no fue sino hasta la década de los 1980 cuando el término "sostenibilidad" comenzó a circular entre técnicos y políticos en las Naciones Unidas. El término aparece en la Estrategia de Conservación Mundial como un concepto clave que aboga por la incorporación de medidas de conservación en los planes de desarrollo (IUCN 1980) (Urry and Macnaghten, 1998).

Una formulación más concreta aparece en el reporte de la Comisión Brundtland titulado Our common future, publicado en 1987 (Redclift, 2005). El reporte lanza el concepto de desarrollo sostenible entendido como una clase de desarrollo que satisface las necesidades del presente "sin comprometer la habilidad de las generaciones futuras para satisfacer sus propias necesidades" (UN, 1987). El reporte de Brundtland introduce por primera vez en el repertorio de la política pública $\mathrm{y}$ del desarrollismo una "perspectiva holística" sobre las relaciones entre naturaleza y actividad humana. En contraste con el conservacionismo clásico, el reporte señala que cualquier intento de protección de la naturaleza que niegue el vínculo con la producción y el mercado, cae en una posición "ingenua" respecto a la protección del ambiente.

A partir del reporte de Brundtland, el término "sostenible" se convierte en uno de los adjetivos preferidos de la palabra desarrollo. El concepto de desarrollo sostenible es el resultado de un esfuerzo por entender y superar las limitaciones de la idea de crecimiento económico, específicamente aquellas limitantes relacionadas con el desarrollo de la tecnología actual, la escasez de recursos sociales y económicos, y la capacidad de la biósfera para absorber los efectos de la actividad humana. De la misma forma, este reporte es un hito en la configuración de un gobierno neoliberal de la naturaleza (Redclift, 2005), pues señala las limitaciones del Estado para responder a los retos del desarrollo sostenible y enfatiza en la importancia de otros actores, específicamente 
de Organizaciones No Gubernamentales y el mercado.

La idea de "desarrollo sostenible" resulta clave en la formulación de la sostenibilidad como un asunto estandarizable. Como Brooks (1992) lo señala, la necesidad de integrar medio ambiente y economía se construyó bajo un repertorio en el cual la actividad económica no es vista como una amenaza para el medio ambiente, sino que, al contrario, el deterioro ambiental es quien la amenaza y el fin último del crecimiento económico es su sostenibilidad indefinida en el tiempo. La economía clásica y la neoclásica han negado los límites que el medio ambiente impone sobre la economía, así como el impacto de la actividad humana en su degradación. El reporte de Brundtland también es un hito en el proceso político de hacer visible el valor de la naturaleza y sus contribuciones a la economía.

A partir de las conferencias y de la producción del reporte, se generó una red de instituciones que fue ganando una posición central en el gobierno de la sostenibilidad y que fue reconocida en la primera conferencia de la Tierra, The Earth Summit, celebrada en Río de Janeiro en 1992. La Conferencia de Río 1992 hizo visibles, como asuntos públicos, la diversidad y los conflictos detrás de la formulación de un marco político para la sostenibilidad. Esta conferencia se pensó como la culminación de un esfuerzo de integración y la apertura de una nueva era ecológica, materializada a través de la Agenda 21. Dicha agenda proponía un conjunto de acciones que mezclaban intervención social y tecnología con el fin de establecer una nueva era de "crecimiento económico". Sin embargo, la discusión de ella hizo explícitas tensiones entre países "industrializados" y en proceso de industrialización, por su participación en un mercado global y por la distribución de riesgos y culpas por los efectos de la producción industrial sobre la biósfera.

Hay algunas contradicciones en las que vale la pena enfatizar:
El concepto de sostenibilidad está ligado a consideraciones más amplias sobre la economía internacional y el libre mercado. Quienes promueven este concepto (ONG y organismos multilaterales) quieren enmarcar la sostenibilidad como prerrequisito a la base de cualquier intercambio económico justo, en tanto benéfico para "todas las partes involucradas". Si bien se reconoce la importancia de proteger los ecosistemas que sostienen la economía global, se afirma que cualquier limitación al libre mercado pone en riesgo el desarrollo sostenible de las naciones.

Así, la integración entre ambiente y economía ha sido un motivo recurrente en el discurso de política pública internacional. Desde su formulación en el reporte de Brundtland y en la Agenda 21 de Río, esta idea ha sido difundida y traducida por académicos y activistas ambientales. Trabajos como el de Pieter Winsemius y Taizo Yakushiji en su libro Beyond Interdependence: The Meshing of the World's Economy and the Earth's Ecology (1991), iniciaron un programa de investigación e intervención para cuantificar el valor de la naturaleza y sus aportes a la economía global en términos de materias y servicios ecosistémicos. Uno de los problemas clave para esta perspectiva es desarrollar herramientas que permitan la medición de las consecuencias de intervenciones específicas: la cuantificación de su impacto. Así mismo, se plantea la necesidad de definir el valor de la naturaleza a través de precios, pues las consecuencias ambientales de la producción a menudo no se reflejan en los precios pagados por bienes y servicios.

En el caso de la producción agrícola, dichas discusiones no han pasado desapercibidas. En el plano local e internacional, diferentes organizaciones han reconocido que la sostenibilidad se ha convertido en un elemento clave que debería organizar la producción agrícola en cultivos como el café o el cacao. La promesa ha sido que la sostenibilidad agrega valor a productos tradicionalmente tratados 
como commodities y comercializados a través de mercados de futuros en bolsas internacionales. En este contexto, por ejemplo, la Federación Nacional de Cafeteros (FNC) desde 2011 ha promovido la producción de cafés sostenibles, como parte de su programa de cafés especiales, mediante el despliegue de una estrategia integral llamada Sustainability matters, que pretende conducir la organización hacia la sustentabilidad. Sin embargo, la idea de la producción sostenible de café es anterior y aparece en los discursos de política cafetera desde fines de los años 1990.

Como la Organización Internacional de Café (OIC) señala en su informe El estado de los cafés sostenibles (2003), un conjunto de iniciativas independientes se insertó gradualmente bajo el paraguas del café sostenible. La mayoría de estas abogaban por cambios específicos en la producción de café y la certificación de mejores prácticas para crear un valor diferencial en el mercado.

La sostenibilidad es un problema mucho más amplio que los tipos de café que se identifican como "sostenibles" per sé [sic] y que se miden en este informe. De hecho, hay una serie de nuevas iniciativas que se distinguen de los cafés orgánicos, de comercio justo y respetuosos con el medio ambiente que también pueden contribuir a la sostenibilidad a largo plazo en el sector del café. Muchas de estas iniciativas se pueden caracterizar como esfuerzos impulsados por la industria que definen la sostenibilidad en algún lugar entre las prácticas convencionales y un nivel mejorado de sostenibilidad que las empresas sienten que pueden alcanzar y pagar razonablemente. Las mejores de estas iniciativas incorporan la verificación de terceros independientes y son muy útiles para los productores, mientras que otras son poco más que tácticas de relaciones públicas. (OIC, 2008, p. 13)
Actualmente, la OIC promueve el uso de esquemas de certificación y verificación como una de las estrategias principales para certificar la producción cafetera como café sostenible. Los esquemas de certificación se perciben como "herramientas poderosas para la adición de valor, acceso a un segmento de mercado de rápido crecimiento y la difusión de buenas prácticas agrícolas, ambientales y sociales" (OIC, 2013). El informe señala que la idea de producir café sostenible se relaciona especialmente con la idea de confianza en la certificación. Mediar dichas relaciones de confianza, es decir, certificar que un café se produce bajo criterios específicos de sostenibilidad es quizá el papel principal de los sellos sostenibles.

El cumplimiento de prácticas "sostenibles" no es visible en el producto. Los esquemas de certificación surgen a medida que actúan como tecnologías de confianza que permiten a los consumidores distinguir las "prácticas" de los productores "honestos". Como resultado de esto, han emergido varios esquemas de estandarización, certificación y verificación que median sociotécnicamente esas relaciones, produciendo dichos mercados.

La OIC también argumenta que los consumidores están cambiando sus patrones de consumo, lo que demuestra un interés creciente en los aspectos económicos, sociales y ambientales de la producción de café. La OIC estima que los cafés sostenibles constituyen el 8 $\%$ de las exportaciones mundiales de café verde y es el segmento de mercado de más rápido crecimiento en los países desarrollados (mercados tradicionales). Las principales iniciativas para café son Fairtrade (Comercio Justo), Organic Certification, UTZ/Rainforest Alliance y Birdfiendly. Adicional a esto, desde empresas privadas han emergido nuevos códigos como iniciativas de verificación sostenibles: el programa CAFE Practices de Starbucks y el programa Nespresso AAA. Así, la idea de sostenibilidad promovida por estos actores es lo suficientemente general como para 
integrar prácticas, objetos y objetivos muy diferentes. Por ejemplo, dentro del marco a través del cual opera el comercio justo, la producción orgánica se considera sostenible. Independientemente de los esquemas de certificación, todos estos productos son percibidos como café sostenible.

\section{¿Café sostenible?}

Más allá de las referencias a las políticas ambientales internacionales, la pregunta sobre qué es o, mejor aún, qué hace que un café sea sostenible, o qué es lo que tiene un café sostenible en sí, prevalece. La OIC señala en el informe El estado de los cafés sostenibles (2003) que la sostenibilidad es un concepto amplio cuyo desarrollo implica acciones más allá de la producción de cafés particulares. La producción de cafés sostenibles es una cuestión de confianza: confiar en el cumplimiento de prácticas "sostenibles" que no son visibles o percibidas en el producto en sí mismo. Una cuestión de confianza es respondida a través de infraestructuras documentales y de auditorías que actúan como tecnologías de confianza. Los esquemas de certificación, por lo tanto, son tecnologías que requieren y producen "relaciones de confianza", entendidas aquí como formas legales privadas que permiten distinguir entre productores honestos que comercializan su café "verdaderamente sostenible" (es decir, bajo un sello de sostenibilidad).

En general, la OIC percibe que el consumidor no tiene claridad sobre los valores agregados diferenciados de los diversos cafés responsables que han emergido. Paradójicamente, esta confusión parece funcional para el mercado cafetero porque, como lo señala la OIC, algunas tiendas y cafés especulan "que los consumidores preferirían no tener que elegir entre comercio orgánico y justo, y un número cada vez mayor de distribuidores importantes desea tener cafés con ambas certificaciones" (ICO, 2003, p. 19). Es común encontrar en el mercado cafés que han adquirido muchas certificaciones: orgánico, comercio justo y Rainforest Alliance en el mismo producto.

Otra "confusión" percibida en relación con los cafés sostenibles es la diferencia que algunos actores cafeteros hacen con respecto a los cafés especiales. El término "café especial" se utiliza para describir los granos de mejor sabor que se producen en microclimas, dentro de unas condiciones de cosecha, de clima, de variedad de café y de procesamiento postcosecha particulares. El término se reserva para café de calidad muy superior reconocida. El término fue usado por primera vez en 1974 por Erna Knutsen en un número de Tea \& Coffee Trade Journal. La evaluación de un café especial se realiza a través de la degustación sistemática del café, siguiendo las pautas definidas por las Asociaciones de Cafés Especiales (SCA en inglés) y que promueven una forma diferente de establecer relaciones comerciales. Un café especial no necesariamente sigue prácticas sostenibles; del mismo modo, un café sostenible no es necesariamente un café especial. Sin embargo, las percepciones y los límites entre estas clasificaciones son muy borrosos. Dado que estos cafés se distinguen de los cafés producidos regularmente, las características especiales de cada uno se perciben como comunes a todos los segmentos alternativos del mercado.

A pesar de estas cuestiones sobre la diferenciación y las múltiples concepciones de sostenibilidad en términos de mercado y políticas, la OIC identifica como cafés sostenibles a los orgánicos, los ecológicos y los certificados para el comercio justo.

Todavía no está claro cuál de los conceptos se convertirá en el estándar general y cuáles permanecerán confinados a nichos. Por su lado, el Centro de Comercio Internacional define los esquemas de certificación como una garantía de que 'las normas y reglamentos de las normas voluntarias se cumplen en un entorno determinado (por ejemplo, 
productor individual, grupo de productores, cooperativa o incluso región)' (ITC, 2012, p. 10)

Para obtener la posibilidad de llevar una etiqueta especial en sus productos, los productores de café "deben cumplir con ciertos requisitos (sociales, económicos, ambientales) y la certificación requiere una confirmación independiente de este estado por parte de un auditor acreditado" (ITC, 2012, p. 12), a renovar anualmente.

\section{¿Estándares para llegar a la sostenibilidad cafetera?}

Los esquemas de certificación (o sellos sostenibles) son presentados como "herramientas poderosas para la adición de valor, acceso a un segmento de mercado de rápido crecimiento y la difusión de buenas prácticas agrícolas, ambientales y sociales" (OIC, 2013). La sostenibilidad en el sector cafetero, como un conjunto de discursos y prácticas, implica el ensamblaje de normas, procesos de auditoría y certificación y comercialización vía sellos. En este sentido, las certificaciones se presentan en informes técnicos, como "instrumentos para monitorear y comunicar la sostenibilidad de los productos basados en los métodos de producción y procesamiento aplicados a lo largo de la cadena de suministro" (Giovanucci y Potts, 2008, p. 4).

También son vistos como un conjunto de criterios para la definición del valor del café y su traducción en precios. Si los productores producen según criterios de sostenibilidad específicos, entonces pueden ser valorados por reglas especiales del mercado. En cierta medida, existe una suposición, organizada materialmente por los estándares, de que estos son productos especializados que pueden diferenciarse ya que "internalizan los costos sociales y ambientales de la producción" (Giovanucci y Potts, 2008, p. 4).

Estas iniciativas sostenibles, en el entendimiento de la OIC, funcionan a fondo en "esquemas de certificación y verificación". En este momento, el mercado cafetero promueve o usa al menos seis iniciativas sostenibles que están directamente relacionadas con la producción de cafés sostenibles: en primer lugar, las certificadoras independientes (Third Party Agencies) como Fairtrade Certified, Organic Certification - Federación Internacional de Movimientos de Agricultura Orgánica (IFOAM), Rainforest Alliance Certified, SMBC "Bird Friendly" y Certificación UTZ Certified del Smithsonian Migratory Bird Center; por otra parte, hay certificaciones desarrolladas por algunas porciones de la cadena del café como productores o tostadores: por ejemplo, el Código Común para la Comunidad del Café (4C). La Asociación 4C es una asociación de membresía abierta que involucra a los productores de café, el comercio y la industria, y la sociedad civil. El código de conducta de 4C abarca una serie de principios sociales, ambientales y económicos para todos los participantes en la cadena de suministro de café verde.

A continuación, se describirán brevemente los principios de dos de los sellos con mayor presencia en el mercado de los sostenibles y que muestran dos tipos de preocupación profundamente entrelazados: la compensación de los costos sociales (Fairtrade) y de los costos ambientales (Rainforest Alliance) de la producción cafetera. Aquí se explica cómo estos dos esquemas de certificación actúan en relación con las promesas en las que construyen su legitimidad. Finalmente, se propone una discusión sobre los límites operativos de los estándares en relación con lo que se puede llamar la paradoja de su integración normativa. Estos dos estándares recrean uno de los principales problemas que estos instrumentos tienen en la transformación material de las formas de producción de café en términos de relaciones sociales más justas con los trabajadores y con la naturaleza: su integración normativa pero su aislamiento respecto a los 
mecanismos de producción de valor de los mercados de gran escala. Para ilustrar este punto, se tratará brevemente el problema del salario mínimo vital desde el punto de vista de los estándares de sostenibilidad.

\section{Lo sostenible como compensación a los costos sociales en la producción cafetera: Fairtrade Certificate.}

Las certificaciones de comercio justo surgieron en los Países Bajos a finales de la década de 1980. La Fundación Max Havelaar lanzó la primera etiqueta de garantía de consumo sobre producción Fairtrade en 1988, para café procedente de México. En el Reino Unido, la Fundación Fairtrade se estableció en 1992, mientras que los primeros productos en llevar la marca Fairtrade fueron lanzados en 1994. La certificación Fairtrade -literalmente mercado justo- se define como una estrategia "para el alivio de la pobreza y el desarrollo sostenible". Como organización, "su propósito es crear oportunidades para los productores y trabajadores que han sido económicamente desfavorecidos o marginados por el sistema de comercio convencional" (FLO, 2013). Se afirma que el acceso justo a los mercados en mejores condiciones comerciales ayudaría a los productores a superar las barreras del desarrollo. Fairtrade es un esquema que garantiza que los agricultores y los trabajadores desfavorecidos de los países en desarrollo obtengan un mejor trato a través del uso de la marca internacional Fairtrade.

En principio, hay dos conjuntos distintos de estándares de comercio justo que construyen dos tipos de productores "desfavorecidos": estándares genéricos de comercio justo para organizaciones de pequeños agricultores $\mathrm{y}$ estándares genéricos de comercio justo para el trabajo contratado. Un conjunto de normas se aplica a los pequeños propietarios que trabajan en cooperativas $u$ otras organizaciones con una estructura democrática, mientras que el otro conjunto se aplica a los trabajadores y propende por salarios decentes, derecho a asociación, salud y seguridad social. Los estándares de comercio justo también cubren los términos de intercambio comercial: la mayoría de los productos tienen un precio de comercio justo, que equivale al valor mínimo que se le debe pagar a los productores por su producto $\mathrm{y}$ trabajo. Además, los productores pueden obtener una suma adicional (Fairtrade Premium) para invertir en proyectos comunitarios a nivel de asociaciones, veredas rurales, y más. En términos prácticos, los esquemas de certificación de comercio justo abarcan un conjunto de criterios tanto ambientales como sociales. Ambos promulgan tipos específicos de actividades como asuntos de administración, que tienen como escenario natural la finca, en este caso, la finca cafetera.

Tabla 1.

Hitos en el desarrollo de FLO (2018)

1997: Fairtrade Labelling Organizations International (FLO) se estableció en Bonn, Alemania, para unir las iniciativas de etiquetado bajo un solo paraguas, y establecer estándares y certificaciones mundiales.

2002: FLO lanza una nueva marca de certificación de comercio justo internacional. Los objetivos del lanzamiento fueron mejorar la visibilidad de la marca en las estanterías de los supermercados, facilitar el comercio transfronterizo y simplificar los procedimientos de exportación tanto para los productores como para los exportadores.

2004: FLO se divide en dos organizaciones independientes. FLO International, que establece los estándares de comercio justo Fairtrade y brinda apoyo comercial al productor, y FLO-CERT, que inspecciona y certifica a las organizaciones de productores y audita a los comerciantes. 
2006: El proceso de armonización de la marca de certificación Fairtrade todavía está en curso. Para entonces todas, excepto tres iniciativas de etiquetado (a saber, Transfair USA, TransFair Canada y Max Havelaar Suiza), han adoptado la nueva marca internacional de certificación Fairtrade. La transición completa a la nueva marca de certificación Fairtrade debería convertirse en realidad, ya que reemplaza gradualmente las marcas de certificación antiguas a diferentes velocidades en varios países.

2007: 21 iniciativas de etiquetado son miembros de FLO International. Las nuevas incorporaciones incluyen México y Australia/Nueva Zelanda.

En Fairtrade, "lo social" se expresa en términos de producción de capacidades organizativas y de gobierno democrático. Por su parte, lo "organizativo" se evidencia mediante el establecimiento de nuevas formas de organización social horizontal (las cooperativas), pero, al mismo tiempo, se hace a través de una "representatividad democrática" dentro de dicha estructura.

Los criterios de desarrollo ambiental en Fairtrade pretenden garantizar que la organización de productores y sus miembros protejan el medio ambiente y hagan de la protección ambiental una parte integral de la gestión de la finca. Existen reglas claras para proteger a los agricultores y trabajadores en relación con el uso de productos químicos, la eliminación de desechos y la protección de los recursos naturales. Esta certificación también prohíbe el uso de organismos modificados genéticamente. Donde sea posible social y económicamente, se alientan a las organizaciones de productores a trabajar hacia prácticas orgánicas. También se espera que minimicen el uso de fertilizantes químicos e insecticidas, y que los cambien gradualmente con fertilizantes naturales y métodos biológicos de control de enfermedades (FLO, 2013).

De manera similar, los criterios respecto a las condiciones laborales de los trabajadores están enmarcados en campos epistémicos y de política más amplios, como los estudios sobre desarrollo y la regulación internacional de trabajo. En los estándares de trabajo contratado, los criterios de desarrollo social garantizan que las empresas reconozcan y apoyen a Fairtrade como un medio para aumentar el empoderamiento y el bienestar de sus trabajadores. Estos estándares

protegen los derechos básicos de los trabajadores tal como se definen en los convenios de la Organización Internacional del Trabajo (OIT) relacionados con: libertad de discriminación (por motivos de raza, religión, género, política y origen étnico o social), libertad de asociación (el derecho a afiliarse a un sindicato) y negociación colectiva de condiciones justas de empleo (salarios, horas de trabajo, horas extraordinarias, pago por enfermedad, etc.) sin trabajo forzado o infantil (edad mínima de 15 años), salud y seguridad ocupacional (un entorno laboral seguro). (FLO, 2013)

El alcance de los estándares de desarrollo ambiental es el mismo para las organizaciones de pequeños agricultores y está destinado a garantizar que las empresas protejan el medio ambiente natural.

Los esquemas de certificación, como dispositivos de mercado, ayudan a promulgar precios diferenciados para el café. En el caso de Fairtrade, hay precios mínimos de comercio justo y una Prima de Comercio Justo. El precio mínimo pagado está determinado por los estándares Fairtrade y se aplica a la mayoría de los productos certificados bajo este sello. Este precio apunta a asegurar que los productores puedan cubrir sus costos promedio de producción de forma sostenible. Este 
mecanismo pretende ser una red de seguridad para los agricultores en los momentos en que los mercados mundiales caen por debajo de un nivel sostenible. Sin esto, los agricultores están completamente a merced del mercado, como ha sido manifiesto en la última crisis de precios.

Cuando el precio de mercado es más alto que el mínimo de comercio justo, el comprador debe pagar el precio más alto. Los productores y comerciantes también pueden negociar precios más altos en función de la calidad y otros atributos. (FLO, 2013)

Además del precio justo, hay un incentivo adicional en dinero: la Prima de Comercio Justo. Este dinero se destina a un fondo comunitario para que los trabajadores y los agricultores lo usen en el mejoramiento de sus condiciones sociales, económicas y ambientales. El uso de la prima se decide

democráticamente por los productores dentro de la organización de agricultores o por los trabajadores de una plantación. La prima se invierte en educación y atención médica, mejoras agrícolas para aumentar el rendimiento y la calidad o instalaciones de procesamiento para aumentar los ingresos. (FLO, 2013)

Para otorgar tales precios, Fairtrade establece una clasificación en relación con la adopción de normas. Este esquema es progresivo también: dentro de cada área hay requisitos mínimos que un productor debe cumplir para ser certificado y requisitos de progreso sobre los cuales la organización certificada debe demostrar una mejora permanente.

Por ejemplo, un requisito mínimo es una prohibición del uso de agroquímicos en la lista de materiales prohibidos de FLO. Un requisito de progreso es la reducción continua en el uso y la toxicidad de los agroquímicos permitidos. De esta manera, las normas permiten a los agricultores más pobres y vulnerables ingresar al sistema, mientras que les ayudan a mejorar gradualmente sus prácticas. Se reconoce que el grado de progreso depende del nivel de beneficios económicos que la organización recibe de Fairtrade y del contexto específico de cada organización. (FLO, 2013)

\section{Protegiendo la naturaleza: Rainforest Alliance Certificate}

Otra de las certificaciones de mayor uso es Rainforest Alliance. La certificación Rainforest Alliance es un conjunto de estándares que las fincas deben cumplir con rigurosidad y que son definidos por la Red de Agricultura Sostenible (RAS). La RAS es una de las coaliciones de ONG más grandes y antiguas enfocadas en el mejoramiento de las condiciones de producción de commodities en los países en desarrollo y en relación con cultivos conectados a la preservación de bosques tropicales. La producción y revisión de normas y políticas son coordinadas por la secretaría de la RAS con sede en San José, Costa Rica. Así mismo, existen organizaciones locales (ahora independientes de la RAS) de certificación y verificación, que auditan y certifican fincas.

Los estándares producidos por este esquema han sido definidos por la secretaría de la RAS, siguiendo los lineamientos de un código de buenas prácticas para establecer estándares sociales y ambientales, de acuerdo con la Alianza Internacional de Acreditación y Etiquetado Social y Ambiental (Iseal en inglés). La Iseal Alliance es una asociación de organizaciones internacionales líderes en certificaciones voluntarias, que establecen estándares y evaluaciones en temas sociales y ambientales. "Esta colaboración representa un importante movimiento global para promover los intereses de los trabajadores, las comunidades y el medio ambiente en el comercio mundial" (Rainforest Alliance, 2013). Estos estándares son definidos por el consenso de un conjunto heterogéneo de actores 
involucrados en cadenas de valor de comercio global e incluye a organismos de inspección, multinacionales, gobiernos, organismos multilaterales y organizaciones de productores.

Tabla 2.

Hitos en el Desarrollo de Rainforest Alliance Certificated (RA, 2019)

\begin{abstract}
1991: Los principios de la agricultura sostenible y la norma de apoyo se desarrollaron por primera vez mediante un proceso que involucró a los principales interesados en América Latina.

1994: Las primeras plantaciones de banano fueron certificadas con base en esta norma. Desde entonces, la norma se ha probado en diferentes granjas de diferentes tamaños, y para muchos otros cultivos y en varios países a través de una serie de auditorías y otras actividades relacionadas con la certificación.

2003: Rainforest Alliance, en su función de la secretaría de la Red de Agricultura Sostenible (RAS), desarrolló una revisión detallada de la versión 2002 de la norma para producir una norma más actualizada, de acuerdo con la misión de la Red.

2003-2004: Se llevaron a cabo consultas públicas durante las cuales se pidió a las organizaciones e individuos de diferentes países que comentaran sobre la norma revisada. Este proceso culminó en una reunión de la Red de Agricultura Sostenible en noviembre de 2004, durante la cual se tomaron las decisiones técnicas finales.

2005: La Red de Agricultura Sostenible aprobó la versión de la norma que llevó a la estructura estándar actual con diez principios.

En 2008, el Comité de Normas Internacionales de la RAS y los equipos técnicos lideraron otro proceso de consulta pública, de acuerdo con los requisitos del Código de Buenas Prácticas de Iseal Alliance para el Establecimiento de Normas Sociales y Ambientales, que dio lugar a la publicación de dos documentos:

- Estándar de Agricultura Sostenible, versión de abril de 2009

- Anexo de SAN: Criterios de SAN adicionales, versión de abril de 2009.
\end{abstract}

Esta red internacional de difusión y auditoría, articulada a actores locales, ha permitido un rápido aumento en el uso de este esquema. Según SAN,

desde 1992, más de 600 certificados para más de 60.000 fincas, incluidas pequeñas granjas familiares de cooperativas y plantaciones, en más de 25 países han cumplido con los estándares SAN en más de 500.000 hectáreas para más de 20 cultivos incluyendo café, cacao, plátano, té, piña, flores y follaje, y cítricos. (Rainforest Alliance, 2013)

Los criterios estándar establecidos por esta certificación son flexibles en términos del porcentaje de cumplimiento para obtenerla $y$ mantenerla. Para ello, las fincas deben cumplir con al menos el $50 \%$ de los criterios aplicables de cada principio y al menos el $80 \%$ de los criterios aplicables totales del estándar de agricultura sostenible. A pesar de este margen de cumplimiento, el estándar es particularmente severo en relación con lo que se define como criterios críticos, es decir, aquellos criterios relacionados con el uso de pesticidas altamente contaminantes o prácticas laborales abiertamente abusivas y explotadoras.

Rainforest Alliance como estándar es particularmente popular en el caso del café, esto debido a que muchas fincas productoras se encuentran en áreas consideradas como de alta prioridad para la conservación. Las zonas de producción cafetera, la selva tropical y los 
países en desarrollo a menudo se superponen. Esta relación es utilizada por Rainforest Alliance como el eje de su estrategia de mercadeo y promoción.

"Rainforest Alliance y sus grupos asociados en la Red de Agricultura Sostenible (RAS) han demostrado que las fincas de café tradicionales y reforestadas son un paraíso para la vida silvestre" (Rainforest Alliance, 2013). Las fincas certificadas sirven como refugio para la vida silvestre $\mathrm{y}$, por lo tanto, los agricultores deben monitorear las especies en las granjas. Esto es particularmente importante para las que están en peligro de extinción y sus hábitats permanecen en tierra. El estándar prescribe que los agricultores deben tomar medidas específicas para protegerlas, esto incluye educar a los trabajadores, prohibir la caza y retirar de sus tierras plantas y animales nocivos para dichas especies. Además, deben proteger los lugares de anidación y liberar la fauna cautiva o registrar animales ante las autoridades correspondientes.

Al igual que en Fairtrade, Rainforest busca reunir dimensiones sociales y ambientales bajo un mismo conjunto de prácticas. La definición de riesgos, su mitigación y la acción local de los productores están asociadas a esta integración. Para SAN, el objetivo de la norma es alentar a las fincas a mitigar los riesgos ambientales y sociales causados por las actividades agrícolas, a través de un proceso de mejora continua.

El estándar se basa en los temas de solidez ambiental, equidad social y viabilidad económica. Las fincas certificadas operan un sistema de gestión ambiental y social de acuerdo con la complejidad de su operación y de acuerdo con la legislación local aplicable. (Rainforest Alliance, 2013)
Los límites operativos de los estándares de sostenibilidad del café: integración normativa y aislamiento de mercado

La integración normativa y el aislamiento de mercado recrean uno de los principales problemas que los estándares tienen en la transformación material de las formas de producción de café en términos de relaciones sociales más justas con los trabajadores y con la naturaleza: su integración normativa, pero su aislamiento respecto a los mecanismos de producción de valor de los mercados de gran escala. Esta limitación es fruto de los marcos interpretativos y de los regímenes de valor de los que hacen parte. Como se ha expuesto al principio de este artículo, los estándares se sitúan como las herramientas adecuadas para la materialización de un concepto neoliberal de desarrollo sostenible, en el que los cambios en la producción agrícola se superponen a las dinámicas del mercado internacional. Los mecanismos del mercado internacional no se negocian, pero en compensación se generan un conjunto de normas y de instrumentos de comunicación entre productores y consumidores que mitigan los impactos negativos de estos mercados.

Para ilustrar este punto, cabe referirse al problema del salario mínimo vital desde el punto de vista de los estándares de sostenibilidad. Ese término hace referencia a aquel salario que cubre de forma digna no solo una canasta básica de alimentos, sino también el acceso a otros elementos que constituyen una vida digna: recreación, educación, vivienda $\mathrm{y}$ ahorro. Este salario vital no corresponde, en términos del monto, necesariamente con el salario mínimo legal de cada país. Los estándares de sostenibilidad normativamente se acogen a las convenciones y recomendaciones de la Organización Internacional del Trabajo para la protección de los impactos sociales de la producción y específicamente a los derechos laborales. 
La Figura 1 muestra una red del ecosistema normativo de estos estándares en este punto. Como es de esperarse, el estándar Fairtrade es el que integra más disposiciones de la OIT, sin embargo, los otros estándares reconocen aquellas disposiciones que cubren los aspectos más generales de protección al trabajo.

Figura 1. Ecosistema normativo sobre protección laboral de los estándares de sostenibilidad Fairtrade. Rainforest Alliance, 4C, INFOAM.

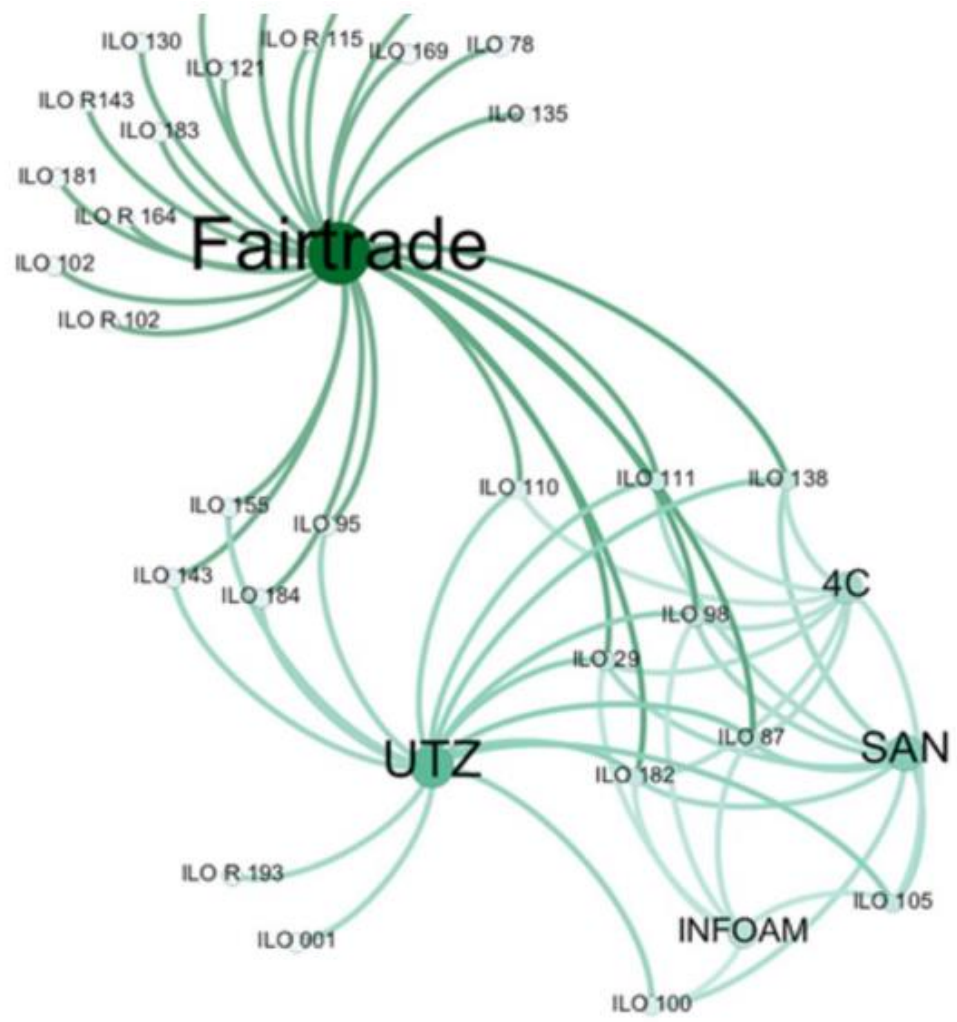

Fuente: Elaboración propia

Esta integración con la normatividad sobre trabajo contrasta con el escaso desarrollo de estrategias y mecanismos de impacto sobre los precios internacionales del café (y en general de las commodities), que son los que a la larga definen la rentabilidad de la producción y la cobertura de los gastos mínimos de operación.

Como se señaló con anterioridad, tanto Fairtrade como Rainforest Alliance definen sus primas con base en el precio internacional del café, definido por instrumentos financieros como el Contrato C de Nueva York. La actual coyuntura de precios históricos bajos hace visible la inoperatividad de los estándares sobre las infraestructuras del mercado internacional. Con los precios actuales, que rozan por debajo de un dólar (USD), las primas de sostenibilidad no alcanzan a cubrir el punto de quiebre de producción y por tanto tienen un impacto nulo en la protección de la calidad del trabajo y en el cubrimiento de un salario mínimo legal. Aquella integración legal y normativa de los estándares contrasta con su impotencia frente a las condiciones y dinámicas del mercado internacional.

\section{A manera de conclusión}

Este artículo muestra el proceso mediante el cual los estándares de sostenibilidad se posicionan en el discurso de política social y económica de los organismos internacionales encargados de la protección del ambiente, y en 
los que representan a los productores de café como la herramienta adecuada para la transformación de los mercados. Los estándares de sostenibilidad responden a una concepción de desarrollo sostenible que permite la protección de la naturaleza y el desarrollo del capitalismo global en términos de comercio internacional. Estos estándares han alcanzado una difusión global bajo la premisa de ofrecer soluciones, basadas en el mercado, a problemas estructurales de las formas contemporáneas de producción agrícola: el impacto nocivo a la naturaleza y la reproducción de relaciones laborales y de mercado abusivas y de explotación.

Este deseo de integración entre economía y sostenibilidad configura el discurso de los certificadores, el cual adquiere un tono holístico en la definición de las rutas de producción agrícola y específicamente de café. Esa integración busca armonizar la producción de ganancias con la protección del medio ambiente y el cuidado de los trabajadores, tal como se despliega en la retórica de Fairtrade y Rainforest. Sin embargo, tal integración supone un costo muy alto: la superposición de estos estándares a dinámicas de mercado históricamente asimétricas, destructivas e injustas, hace operativo el estándar en un sentido de gestión documental, pero tiene muy poco margen de maniobra para la transformación material de las ecologías de la producción de café.

En futuros trabajos es necesaria una exploración multisituada de los estándares, mostrando las diferentes localidades que coordinan y las traducciones entre dominios de práctica. De dicha multiplicidad y fragmentación pueden emerger sensibilidades alternativas para entender los procesos de estandarización en su complejidad (Lampland y Leigh Star, 2011). Pensar en la producción de entidades/mercados, como los cafés sostenibles, a través de esquemas de certificación, significa rastrear dichos objetos en diversos espacios y localidades. Una localidad importante son las fincas cafeteras, pues estas casi siempre son imaginadas por actores que, como los estandarizadores de Iseal, se mueven entre imaginarios coloniales y poscoloniales de la vida campesina en el sur global, mientras promueven formas de organización que tienen como modelo la firma y un acercamiento administrativo a la finca, ante todo como unidad productiva.

Finalmente, las políticas y los mercados que se materializan a través de los estándares de sostenibilidad pueden imaginarse de múltiples formas y desde espacios diversos, tales como aquellos propios de la producción cafetera. $\mathrm{Si}$ los estándares, a pesar de sus fragmentaciones, generan interdependencias de diversos actores produciendo mercados sostenibles, es porque es en las fincas cafeteras donde se materializan dichos compromisos e interdependencias. Retomando a Hichcliffe (2007) "Solo al trabajar con la multiplicidad de cosas, prestando la debida atención a las ecologías de acción que hacen que las cosas sucedan, se puede decir que una política es sostenible" ( $p$. 34).

\section{Referencias}

Bain, C., Ransom, E., y Higgins, V. (2013). Private Agri-Food Standards: Contestation, Hybridity and the Politics of Standards. International Journal of Sociology of Agriculture and Food, 20(1), 1-10.

Bain, C. (2010) Structuring the Flexible and Feminized Labor Market: GlobalGAP Standards for Agricultural Labor in Chile. Signs, 35(2), 343-370. https://www.jstor.org/stable/10.1086/605 479.

Bain, C. y Dandachi, T. (2014). Governing GMOs: The (Counter)Movement for Mandatory and Voluntary Non-GMO Labels. Sustainability, 6(12), 9456-9476. 
Bain, C. y M. Hatanaka. (2010). The Practice of Third-Party Certification: Enhancing Environmental Sustainability and Social Justice in the Global South? In V. Higgins and W. Larner (eds). Calculating the Social: Standards and the Reconfiguration of Governing [pp. 56-74]. Basingstoke: Palgrave MacMillan.

Bain, C. y Selfa. T. (2017). Non-GMO vs Organic Labels: Purity or Process Standards in a GMO Contaminated Landscape. Agriculture and Human Values.

Barham, B. y Weber, J. (2012). The Economic Sustainability of Certified Coffee: Recent Evidence from Mexico and Peru. World Development, 40(6), 1269-1279

Bowker, G. y Star, S.L. (1999) Sorting things out. Classification and its consequences. London: MIT Press.

Brooks, D. (1992) The challenge of sustainability: Is integrating environment and economy enough? Policy Sciences, $25,401-408$.

Busch, L. (2010) Can Fairy Tales Come True? The Surprising Story of Neoliberalism and World Agriculture, Sociologia Ruralis, 50(4)

Busch, L. (2011) Standards: Recipes for Reality. London: MIT Press.

Hatanaka, M. 2010. Trust, Certification, and Partnership in an Organic Shrimp Network: Rethinking Transnational Alternative Agrifood Networks. World Development 38(5),706-716.

Hichliffe, S. (2007) Geographies of Nature. Societies environments ecologies. London: Sage.
ICO, International Coffee Organization (2013) History http://www.ico.org/icohistory_e.asp?secti on=About_Us

Lampland, M y Leigh Star, S. (2009) Standards and Their Stories: How Quantifying, Classifying, and Formalizing Practices Shape Everyday Life. Ithaca: Ithaca University Press.

Law, J. y Urry, J. (2004) Enacting the social, Economy and Society, 33(3), 390-410, DOI: $10.1080 / 0308514042000225716$

Loconto, A. y Fouilleux, E. (2014). Politics of private regulation: ISEAL and the shaping of transnational sustainability governance. Regulation \& Governance, 8(2), 166-185.

Loconto, M y Busch, L (2010). Standards, techno-economic networks and playing fields: Performing the global economy. Review of International Political Economy. 3, 507, 536

MacNeill, J., Winsemius, P. y Yakushiji, T. 1991. Beyond Interdependence. The Meshing of the World's Economy and the Earth's Ecology. Oxford: Oxford University Press.

Maertens, M. (2016). Private Sustainability Standards in the Ugandan Coffee Sector: Empty Promises or Catalysts for Development? 235004.

Pinto, Gardner, Mcdermott y Ayub. (2014). Group certification supports an increase in the diversity of sustainable agriculture network-rainforest alliance certified coffee producers in Brazil. Ecological Economics, 107, 59-64.

Redclift, M. (2005) Sustainable Development (1987-2005): An Oxymoron Comes of 
Age, Sustainable Development 13(4), 212-227.

Renard, M. (2014) In the name of Conservation: CAFE Practices and Fair Trade in México. Journal of Business Ethics, 92, 287-299.

Rueda X y Lambin E. (2014) Global agriculture and land use changes in the 21st century: Achieving a balance between food security, urban diets and nature conservation. The Evolving Sphere of Food Security (ISBN 978-0-19-9354061)

Thévenot, L. (2009). Governing Life by Standards: A View from Engagements. Social Studies of Science 39(5): 793-813.

Timmermans, S. y Epstein, S. (2010). A world of standards but not a standard world: Toward a sociology of standards and standardization. Annual Review of Sociology, 36, 69-89.

UN, United Nations. (1987) Report of the World Commission on Environment and Development Our Common Future. http://conspect.nl/pdf/Our_Common_Fut ure-Brundtland_Report_1987.pdf
UN, United Nations. (1992) United Nations Conference on Environment \& Development Río de Janeiro, Brazil, 3 to 14 June 1992. AGENDA 21. http://sustainabledevelopment.un.org/cont ent/documents/Agenda21.pdf

UN, United Nations. (2002) Report of the World Summit on Sustainable Development. Johannesburg, South Africa, 26 August- 4 September 2002 http://www.un.org/jsummit/html/docume nts/summit_docs/131302_wssd_report_re issued.pdf

Urry, J. y Macnaghten, P. (1998) Contested Natures. London: Sage.

Van Der Kamp, M. (2012) Coexisting Organic Standards: Setting Boundaries, Navigating Multiplicity and Enacting Commonality. En M. Barbier and B. Elzen (Eds.), System Innovations, Knowledge Regimes, and Design Practices towards Transitions for Sustainable Agriculture. Inra [online].

Woolgar, S., Coopman, C. y Neyland, D. (2009). Does STS Mean Business? Organization. 16(1), 5-30 